\title{
Comparison of the Bayesian Methods on Interval-Censored Data for Weibull Distribution
}

\author{
Al Omari Mohammed Ahmed \\ Department of Mathematics, Faculty of Arts and Science in Qilwah, Al Baha University, Baha, KSA \\ Email: alomari1050@hotmail.com
}

Received 19 May 2014; revised 15 June 2014; accepted 30 June 2014

Copyright (C) 2014 by author and Scientific Research Publishing Inc.

This work is licensed under the Creative Commons Attribution International License (CC BY).

http://creativecommons.org/licenses/by/4.0/

(c) (i) Open Access

\section{Abstract}

This study considers the estimation of Maximum Likelihood Estimator and the Bayesian Estimator of the Weibull distribution with interval-censored data. The Bayesian estimation can't be used to solve the parameters analytically and therefore Markov Chain Monte Carlo is used, where the full conditional distribution for the scale and shape parameters are obtained via Metropolis-Hastings algorithm. Also Lindley's approximation is used. The two methods are compared to maximum likelihood counterparts and the comparisons are made with respect to the mean square error (MSE) to determine the best for estimating of the scale and shape parameters.

\section{Keywords}

Weibull Distribution, Bayesian Method, Interval Censored, Metropolis-Hastings Algorithm, Lindley's Approximation

\section{Introduction}

The Weibull distribution observably has the widest variety of applications in many areas, including life testing, reliability theory and others. The most used methods, which are considered to be the traditional methods, are maximum likelihood and the moment estimation (Cohen and Whitten, [1]). Sinha [2] estimated the parameters of Weibull distribution by maximum likelihood and Bayesian using Lindley’s approximation. Smith [3] developed the maximum likelihood and Bayesian estimators and compared them using the three-parameter Weibull distribution. Singh et al. [4] used the Bayesian estimation approach to estimate the parameters of exponentiated Weibull. Hossain and Zimmer [5] estimated the scale and shape parameters of Weibull distribution using complete and censored samples by maximum likelihood estimator and least squares method. Nassar and Eissa [6] 
obtained the Bayesian approach using Lindely approximations to estimate the two shape parameters and the reliability function of the exponentiated Weibull distribution. Soliman et al. [7] estimated Weibull distribution by using maximum likelihood estimator and Bayesian approach followed by estimating the hazard and reliability functions. Kantar and Senoglu [8] reported their findings on the comparative study for the location and scale parameters of the Weibull distribution with a given shape parameter. Gupta et al. [9] estimated Weibull extension model by Bayesian method using Markov Chain Monte Carlo (MCMC). Kundu and Howlader [10] obtained Bayesian inference and prediction of the inverse Weibull distribution for Type-II censored data, where the Gibbs sampling technique was used to generate MCMC samples from the posterior distribution followed by an importance sampling technique for constructing the Bayes estimation. Comparison between Bayesian and maximum likelihood estimation of the scale parameter in Weibull distribution with known shape was considered by Pandey et al. [11].

\section{Methodology}

\subsection{Maximum Likelihood Estimation of Weibull Distribution with Interval-Censored Data}

The probability density function of Weibull distribution is:

$$
f(x ; \lambda, \alpha)=\frac{\alpha}{\lambda} x^{\alpha-1} \exp \left(-\frac{x^{\alpha}}{\lambda}\right)
$$

The cumulative distribution function (cdf) of the Weibull distribution is given as

$$
F(x ; \lambda, \alpha)=1-\exp \left(-\frac{x^{\alpha}}{\lambda}\right),
$$

with the scale parameter $\lambda$ and the shape parameter $\alpha$ of the Weibull distribution

The likelihood function of interval censored as given in Flygare et al. [12] is

$$
\begin{aligned}
L\left(\lambda, \alpha \mid l_{i}, u_{i}\right) & =\prod_{i=1}^{n}\left[F\left(u_{i} ; \lambda, \alpha\right)-F\left(l_{i} ; \lambda, \alpha\right)\right]=\prod_{i=1}^{n}\left[\left(1-\exp \left(-\frac{u_{i}^{\alpha}}{\lambda}\right)\right)-\left(1-\exp \left(-\frac{l_{i}^{\alpha}}{\lambda}\right)\right)\right] \\
& =\prod_{i=1}^{n}\left[\exp \left(-\frac{l_{i}^{\alpha}}{\lambda}\right)-\exp \left(-\frac{u_{i}^{\alpha}}{\lambda}\right)\right] .
\end{aligned}
$$

The logarithm of the likelihood function with interval censored can be expressed as follows:

$$
\ln L\left(\lambda, \alpha \mid l_{i}, u_{i}\right)=\sum_{i=1}^{n} \log \left[\exp \left(-\frac{l_{i}^{\alpha}}{\lambda}\right)-\exp \left(-\frac{u_{i}^{\alpha}}{\lambda}\right)\right]
$$

To obtain the equations for the unknown parameters, we differentiate Equation (2) partially with respect to the scale and shape parameters and equal it to zero. The resulting equations are given respectively as,

$$
\begin{gathered}
\frac{\partial L\left(\lambda, \alpha \mid l_{i}, u_{i}\right)}{\partial \lambda_{i}}=\sum_{i=1}^{n} \frac{l_{i}^{\alpha}}{\lambda^{2}} \exp \left(-\frac{l_{i}^{\alpha}}{\lambda}\right)-\frac{u_{i}^{\alpha}}{\lambda^{2}} \exp \left(-\frac{u_{i}^{\alpha}}{\lambda}\right) / D_{i} \\
\frac{\partial L\left(\lambda, \alpha \mid l_{i}, u_{i}\right)}{\partial \alpha_{i}}=\sum_{i=1}^{n} \frac{u_{i}^{\alpha}}{\lambda} \ln \left(u_{i}\right) \exp \left(-\frac{u_{i}^{\alpha}}{\lambda}\right)-\frac{l_{i}^{\alpha}}{\lambda} \ln \left(l_{i}\right) \exp \left(-\frac{l_{i}^{\alpha}}{\lambda}\right) / D_{i}
\end{gathered}
$$

where

$$
D_{i}=\exp \left(-\frac{l_{i}^{\alpha}}{\lambda}\right)-\exp \left(-\frac{u_{i}^{\alpha}}{\lambda}\right)
$$

cannot be solved analytically, and for that we employed Newton Raphson method to find the numerical solution. 


\subsection{Bayesian Using Gamma Prior Estimation of Weibull Based on Interval-Censored Data}

In this subsection we consider the case when both the scale and shape parameters are unknown, and we compute the Bayes estimates of the shape and scale parameters. It is assumed that $\lambda$ and $\alpha$ each have independent gamma $(a, b)$, and gamma $(c, d)$ priors respectively

$$
\begin{aligned}
& \pi_{1}(\lambda / a, b)=\lambda^{a-1} \exp (-b \lambda) \\
& \pi_{2}(\alpha / c, d)=\alpha^{c-1} \exp (-d \alpha)
\end{aligned}
$$

It is a natural conjugate prior.

The posterior probability density function of $\lambda$ and $\alpha$ given the data with gamma prior is combining Equation (7) with likelihood function and using Bayes theorem, the joint posterior distribution is derived as see $\mathrm{Al}$ Omari et al. [13]

$$
\begin{aligned}
\prod_{1}\left(\lambda, \alpha \mid l_{i}, u\right) & =\frac{L\left(\lambda, \alpha \mid l_{i}, u_{i}\right) \pi_{1}(\lambda / a, b) \pi_{2}(\alpha / c, d)}{\int_{0}^{\infty} \int_{0}^{\infty} L\left(\lambda, \alpha \mid l_{i}, u_{i}\right) \pi_{1}(\lambda / a, b) \pi_{2}(\alpha / c, d) \mathrm{d} \lambda \mathrm{d} \alpha} \\
& =\frac{\prod_{i=1}^{n}\left[\exp \left(-l_{i}^{\alpha} / \lambda\right)-\exp \left(-u_{i}^{\alpha} / \lambda\right)\right] \lambda^{a-1} \alpha^{c-1} \exp (-(b \lambda+d \alpha))}{\int_{0}^{\infty} \int_{0}^{\infty} \prod_{i=1}^{n}\left[\exp \left(-l_{i}^{\alpha} / \lambda\right)-\exp \left(-u_{i}^{\alpha} / \lambda\right)\right] \lambda^{a-1} \alpha^{c-1} \exp (-(b \lambda+d \alpha)) \mathrm{d} \lambda \mathrm{d} \alpha} .
\end{aligned}
$$

With this, the Bayes estimates for the scale and shape parameters under squared error loss function are given as:

$$
\begin{aligned}
\hat{\lambda}= & \frac{\int_{0}^{\infty} \int_{0}^{\infty} \lambda^{a} \alpha^{c-1} \prod_{i=1}^{n}\left[\exp \left(-l_{i}^{\alpha} / \lambda\right)-\exp \left(-u_{i}^{\alpha} / \lambda\right)\right] \exp (-(b \lambda+d \alpha)) \mathrm{d} \lambda \mathrm{d} \alpha}{\int_{0}^{\infty} \int_{0}^{\infty} \prod_{i=1}^{n}\left[\exp \left(-l_{i}^{\alpha} / \lambda\right)-\exp \left(-u_{i}^{\alpha} / \lambda\right)\right] \lambda^{a-1} \alpha^{c-1} \exp (-(b \lambda+d \alpha)) \mathrm{d} \lambda \mathrm{d} \alpha} . \\
\hat{\alpha}= & \frac{\int_{0}^{\infty} \int_{0}^{\infty} \lambda^{a-1} \alpha^{c} \prod_{i=1}^{n}\left[\exp \left(-l_{i}^{\alpha} / \lambda\right)-\exp \left(-u_{i}^{\alpha} / \lambda\right)\right] \exp (-(b \lambda+d \alpha)) \mathrm{d} \lambda \mathrm{d} \alpha}{\int_{0}^{\infty} \int_{0}^{n} \prod_{i=1}^{n}\left[\exp \left(-l_{i}^{\alpha} / \lambda\right)-\exp \left(-u_{i}^{\alpha} / \lambda\right)\right] \lambda^{a-1} \alpha^{c-1} \exp (-(b \lambda+d \alpha)) \mathrm{d} \lambda \mathrm{d} \alpha} .
\end{aligned}
$$

The integration of the scale and shape parameter we can't solve it analytical for that we used MetropolisHastings algorithm and Lindley's approximation to solve the problem.

\section{Metropolis-Hastings Algorithm}

The Metropolis-Hastings algorithm is a very general Markov Chain Mote Carlo method, it can be used to obtain random samples from any arbitrarily complicated target distribution of any dimension that is known up to a normalizing constant. In fact, Metropolis algorithm is an alternative to Gibbs sampler that does not require availability of full conditionals see Hastings [14] and Soliman et al. [15].

Therefore, the full conditional of the posterior density function using gamma prior of $\lambda$ and $\alpha$ given the data are combining the gamma prior with likelihood as given below

$$
\Pi\left(\lambda, \alpha \mid l_{i}, u_{i}\right) \propto \lambda^{a-1} \alpha^{c-1} \prod_{i=1}^{n}\left[\exp \left(-l_{i}^{\alpha} / \lambda\right)-\exp \left(-u_{i}^{\alpha} / \lambda\right)\right] \exp (-(b \lambda+d \alpha)) .
$$

From Equation (11) we can get the conditional posterior of the scale parameter $\lambda$ as follows

$$
\Pi\left(\lambda \mid \alpha ; l_{i}, u_{i}\right) \propto \prod_{i=1}^{n}\left[\exp \left(-l_{i}^{\alpha} / \lambda\right)-\exp \left(-u_{i}^{\alpha} / \lambda\right)\right] \lambda^{a-1} \exp (-b \lambda) .
$$

The conditional posterior of the shape parameter $\alpha$ is given below 


$$
\Pi\left(\alpha \mid \lambda ; l_{i}, u_{i}\right) \propto \prod_{i=1}^{n}\left[\exp \left(-l_{i}^{\alpha} / \lambda\right)-\exp \left(-u_{i}^{\alpha} / \lambda\right)\right] \alpha^{c-1} \exp (-d \alpha)
$$

As show in the conditional posterior of the scale and shape parameters it's not follow any close distribution for that we suggest to use the Metropolis-Hastings algorithm to generate MCMC sample.

\section{Algorithm:}

1) Start with initial value $\lambda_{0}, \alpha_{0}$.

2) The current value $\lambda_{i}, \alpha_{i}$ and generate the candidate value $\lambda^{*}, \alpha^{*}$ from arbitrary distribution uniform $(0,1)$.

3) The next value of $\lambda_{i}$ is given below as

where

$$
\lambda_{i+1}=\left\{\begin{array}{l}
\lambda^{*} \text { with probability } p \\
\lambda_{i} \text { with probability } 1-p
\end{array}\right.
$$

$$
p=\min \left\{1, \frac{\Pi\left(\lambda^{*} \mid \alpha ; l_{i}, u_{i}\right)}{\Pi\left(\lambda_{i} \mid \alpha ; l_{i}, u_{i}\right)}\right\}
$$

4) Generate $u$ from Uniform $(0,1)$ and accept $\lambda^{*}$ with probability $p$ if $\lambda^{*}<p$ and return to step 2), otherwise accept $\lambda_{i}$.

5) The next value of $\alpha_{i}$ is given below as

where

$$
\alpha_{i+1}=\left\{\begin{array}{l}
\alpha^{*} \text { with probability } p \\
\alpha_{i} \text { with probability } 1-p
\end{array}\right.
$$

$$
p=\min \left\{1, \frac{\Pi\left(\alpha^{*} \mid \lambda ; l_{i}, u_{i}\right)}{\Pi\left(\alpha_{i} \mid \lambda ; l_{i}, u_{i}\right)}\right\}
$$

6) Generate $u$ from uniform $(0,1)$ and accept $\alpha^{*}$ with probability $p$ if $\alpha^{*}<p$ and return to step 2), otherwise accept $\alpha_{i}$ and return to step 2).

7) The Bayesian based interval-censored data of the scale and shape parameters under the squared error loss function is given as

$$
\begin{aligned}
& \hat{E}_{1}\left(\lambda \mid \alpha ; l_{i}, u_{i}\right)=\frac{1}{n} \sum_{i=1}^{n} \lambda_{i} \\
& \hat{E}_{2}\left(\alpha \mid \lambda ; l_{i}, u_{i}\right)=\frac{1}{n} \sum_{i=1}^{n} \alpha_{i} .
\end{aligned}
$$

8) Obtain the posterior variance of Bayesian using gamma prior based interval-censored data as

$$
\begin{aligned}
& \hat{V_{1}}\left(\lambda \mid \alpha ; l_{i}, u_{i}\right)=\frac{1}{n} \sum_{i=1}^{n}\left(\lambda_{i}-\hat{E}\left(\lambda \mid \alpha ; l_{i}, u_{i}\right)\right)^{2} \\
& \hat{V}_{2}\left(\alpha \mid \lambda ; l_{i}, u_{i}\right)=\frac{1}{n} \sum_{i=1}^{n}\left(\alpha_{i}-\hat{E}\left(\alpha \mid \lambda ; l_{i}, u_{i}\right)\right)^{2} .
\end{aligned}
$$

\subsection{Lindley's Approximation}

The Equations (9) and (10) cannot be solved analytically and for that we obtained Lindley's expansion to solve the parameters approximation.

According to Al Omari et al. [16] Lindley’s approximation proposed a ratio of integral of the form

$$
\int w(\lambda) \exp \{L(\lambda)\} \mathrm{d} \lambda / \int v(\lambda) \exp \{L(\lambda)\} \mathrm{d} \lambda
$$

where $L(\lambda)$ is the log-likelihood and $w(\lambda), v(\lambda)$ are arbitrary functions of $\lambda$ in applying this procedure, 
it is assumed that $v(\lambda)$ is the prior distribution for $\lambda$ and $w(\lambda)=u(\lambda) \cdot v(\lambda)$ with $u(\lambda)$ being some function of interest.

The posterior expectation according to Sinha [2] is

$$
E(u(\lambda) \mid t)=\int v(\lambda) \exp \{L(\lambda)+\rho(\lambda)\} \mathrm{d} \lambda / \int \exp \{L(\lambda)+\rho(\lambda)\} \mathrm{d} \lambda
$$

where

$$
\rho=\log (v(\lambda))
$$

According to [1] Lindley expansion is therefore approximated asymptotically by

$$
E(u(\lambda) \mid t)=u+\frac{1}{2}\left(u_{11} \sigma_{11}+u_{22} \sigma_{22}\right)+u_{1} \rho_{1} \sigma_{11}+u_{2} \rho_{2} \sigma_{22}+\frac{1}{2}\left(L_{30} u_{1} \sigma_{11}^{2}+L_{03} u_{2} \sigma_{22}^{2}\right)
$$

where $L$ is the log-likelihood equation in (2). See Sinha [2] for more detail.

Taking the scale parameter $\lambda$ estimation, where

$$
\begin{aligned}
& \rho=\ln \left(\pi_{1}(\lambda / a, b)\right)+\ln \left(\pi_{2}(\alpha / c, d)\right), \quad \rho_{1}=\frac{\partial \rho}{\partial \lambda}=\frac{a-1}{\lambda}-b, \rho_{2}=\frac{\partial \rho}{\partial \alpha}=\frac{c-1}{\alpha}-d \\
& u=\lambda, \quad u_{1}=\frac{\partial u}{\partial \lambda}=1, \quad u_{2}=u_{11}=u_{22}=0
\end{aligned}
$$

For the shape parameter

$$
\begin{aligned}
& u=\alpha, u_{2}=\frac{\partial u}{\partial \alpha}=1, u_{1}=u_{11}=u_{22}=0 \\
& \sigma_{11}=\left(-L_{20}\right)^{-1}, \quad \sigma_{22}=\left(-L_{02}\right)^{-1} \\
& L_{20}=\frac{\partial^{2} L}{\partial \lambda^{2}}=\sum_{i=1}^{n}-\frac{2 l_{i}^{\alpha} \mathrm{e}^{-\frac{l_{i}^{\alpha}}{\lambda}}}{\lambda^{3}}+\frac{\left(l_{i}^{\alpha}\right)^{2} \mathrm{e}^{-\frac{l_{i}^{\alpha}}{\lambda}}}{\lambda^{4}}+\frac{2 u_{i}^{\alpha} \mathrm{e}^{-\frac{u_{i}^{\alpha}}{\lambda}}}{\lambda^{3} D_{i}}-\frac{\left(u_{i}^{\alpha}\right)^{2} \mathrm{e}^{-\frac{u_{i}^{\alpha}}{\lambda}}}{\lambda^{4} D_{i}}+\frac{u_{i}^{\alpha} \mathrm{e}^{-\frac{u_{i}^{\alpha}}{\lambda}}\left(l_{i}^{\alpha} \mathrm{e}^{-\frac{l_{i}^{\alpha}}{\lambda}}-u_{i}^{\alpha} \mathrm{e}^{-\frac{u_{i}^{\alpha}}{\lambda}}\right)}{D_{i}^{2}} \\
& L_{02}=\frac{\partial^{2} L}{\partial \alpha^{2}}=\sum_{i=1}^{n} \frac{u_{i}^{\alpha} \ln \left(u_{i}\right)^{2} \mathrm{e}^{-\frac{u_{i}^{\alpha}}{\lambda}}}{\lambda}-\frac{\left(u_{i}^{\alpha}\right)^{2} \ln \left(u_{i}\right)^{2} \mathrm{e}^{-\frac{u_{i}^{\alpha}}{\lambda}}}{\lambda^{2}}-\frac{l_{i}^{\alpha} \ln \left(l_{i}\right)^{2}}{\lambda}+\frac{u_{i}^{\alpha} \ln \left(u_{i}\right) \mathrm{e}^{-\frac{u_{i}^{\alpha}}{\lambda}}}{\lambda} \\
& L_{30}=\frac{\partial^{3} L}{\partial \lambda^{3}}=\sum_{i=1}^{n} \frac{6 l_{i}^{\alpha} \mathrm{e}^{-\frac{l_{i}^{\alpha}}{\lambda}}}{\lambda^{4}}-\frac{6\left(l_{i}^{\alpha}\right)^{2} \mathrm{e}^{-\frac{l_{i}^{\alpha}}{\lambda}}}{\lambda^{5}}+\frac{\left(l_{i}^{\alpha}\right)^{3} \mathrm{e}^{-\frac{l_{i}^{\alpha}}{\lambda}}}{\lambda^{6}}+\frac{6 u_{i}^{\alpha} \mathrm{e}^{-\frac{u_{i}^{\alpha}}{\lambda}}}{\lambda^{4} D_{i}}+\frac{6\left(u_{i}^{\alpha}\right)^{2} \mathrm{e}^{-\frac{u_{i}^{\alpha}}{\lambda}}}{\lambda^{5} D_{i}}-\frac{4 u_{i}^{\alpha} \mathrm{e}^{-\frac{u_{i}^{\alpha}}{\lambda}}\left(l_{i}^{\alpha} \mathrm{e}^{-\frac{l_{i}^{\alpha}}{\lambda}}-u_{i}^{\alpha} \mathrm{e}^{-\frac{u_{i}^{\alpha}}{\lambda}}\right)}{\lambda D_{i}^{2}} \\
& -\frac{\left(u_{i}^{\alpha}\right)^{3} \mathrm{e}^{-\frac{u_{i}^{\alpha}}{\lambda}}}{\lambda^{6} D_{i}}+\frac{2\left(u_{i}^{\alpha}\right)^{2} \mathrm{e}^{-\frac{u_{i}^{\alpha}}{\lambda}}\left(l_{i}^{\alpha} \mathrm{e}^{-\frac{l_{i}^{\alpha}}{\lambda}}-u_{i}^{\alpha} \mathrm{e}^{-\frac{u_{i}^{\alpha}}{\lambda}}\right)}{\lambda^{2} D_{i}^{2}}-\frac{2 u_{i}^{\alpha} \mathrm{e}^{-\frac{u_{i}^{\alpha}}{\lambda}}\left(l_{i}^{\alpha} \mathrm{e}^{-\frac{l_{i}^{\alpha}}{\lambda}}-u_{i}^{\alpha} \mathrm{e}^{-\frac{u_{i}^{\alpha}}{\lambda}}\right)^{2}}{D_{i}^{3}} \\
& +\frac{1}{\lambda^{2} D_{i}^{2}}\left(u_{i}^{\alpha} \mathrm{e}^{-\frac{u_{i}^{\alpha}}{\lambda}}\left(-\frac{2 l_{i}^{\alpha} \mathrm{e}^{-\frac{l_{i}^{\alpha}}{\lambda}}}{\lambda^{3}}+\frac{\left(l_{i}^{\alpha}\right)^{2} \mathrm{e}^{-\frac{l_{i}^{\alpha}}{\lambda}}}{\lambda^{4}}+\frac{2 u_{i}^{\alpha} \mathrm{e}^{-\frac{u_{i}^{\alpha}}{\lambda}}}{\lambda^{3}}-\frac{\left(u_{i}^{\alpha}\right)^{2} \mathrm{e}^{-\frac{u_{i}^{\alpha}}{\lambda}}}{\lambda^{4}}\right)\right) \\
& L_{03}=\frac{\partial^{3} L}{\partial \alpha^{3}}=\sum_{i=1}^{n} \frac{u_{i}^{\alpha} \ln \left(u_{i}\right)^{3} \mathrm{e}^{-\frac{u_{i}^{\alpha}}{\lambda}}}{\lambda}-\frac{3\left(u_{i}^{\alpha}\right)^{2} \ln \left(u_{i}\right)^{3} \mathrm{e}^{-\frac{u_{i}^{\alpha}}{\lambda}}}{\lambda^{2}}+\frac{\left(u_{i}^{\alpha}\right)^{3} \ln \left(u_{i}\right)^{3} \mathrm{e}^{-\frac{u_{i}^{\alpha}}{\lambda}}}{\lambda^{3}}-\frac{l_{i}^{\alpha} \ln \left(l_{i}\right)^{3}}{\lambda} \\
& +\frac{u_{i}^{\alpha} \ln \left(u_{i}\right)^{2} \mathrm{e}^{-\frac{u_{i}^{\alpha}}{\lambda}}}{\lambda}-\frac{\left(u_{i}^{\alpha}\right)^{2} \ln \left(u_{i}\right)^{2} \mathrm{e}^{-\frac{u_{i}^{\alpha}}{\lambda}}}{\lambda^{2}} .
\end{aligned}
$$




\section{Simulation Study}

To assess the performance of the maximum likelihood and Bayesian with help of the Lindley's approximation and Markov Chain Monte Carlo, where the Metropolis-Hastings algorithm used to estimate the scale and shape parameters, the mean squared errors (MSE) for each method were calculated using 10,000 replications for sample size $n=25,50$ and 100 of Weibull distribution with interval-censored data for different value of parameters were the scale parameter $\lambda=2$, shape parametric $\alpha=0.5,1,1.5$ and 2 , the considered values of $\lambda, \alpha$ are meant for illustration only and other values can also be taken for generating the samples from Weibull distribution.

\section{Discussion}

As shown in Table 1, the estimate of the scale parameter $\lambda$ of Weibull distribution with interval-censored data was compared between maximum likelihood (MLE), Bayesian using Lindley’s approximation (BL) and Bayesian using Metropolis-Hastings algorithm (BM) by mean squared error (MSE). We observed that the Bayesian using

Table 1. Estimated average lengths of the scale parameter and MSE of Weibull distribution.

\begin{tabular}{ccccc}
\hline Size & Estimators & MLE & BJ & BM \\
\hline & $\alpha=0.5$ & $1.8918(0.0331)$ & $1.8891(0.0340)$ & $1.8901(0.0335)$ \\
25 & $\alpha=1$ & $1.9151(0.0312)$ & $1.9041(0.0322)$ & $1.9241(0.0301)$ \\
& $\alpha=1.5$ & $1.9255(0.0319)$ & $1.9137(0.0325)$ & $1.9308(0.0311)$ \\
& $\alpha=2$ & $1.9368(0.0307)$ & $1.9418(0.0301)$ & $1.9455(0.0299)$ \\
& $\alpha=0.5$ & $1.9018(0.0318)$ & $1.8986(0.0331)$ & $1.9128(0.0327)$ \\
& $\alpha=1$ & $1.9255(0.0289)$ & $1.9114(0.0300)$ & $1.9311(0.0272)$ \\
& $\alpha=1.5$ & $1.9314(0.0271)$ & $1.9281(0.0299)$ & $1.9400(0.0260)$ \\
& $\alpha=2$ & $1.9417(0.0265)$ & $1.9501(0.0257)$ & $1.9522(0.0259)$ \\
& $\alpha=0.5$ & $1.9161(0.0300)$ & $1.8991(0.0321)$ & $1.9211(0.0310)$ \\
& $\alpha=1$ & $1.9366(0.0276)$ & $1.9213(0.0288)$ & $1.9441(0.0265)$ \\
& $\alpha=1.5$ & $1.9443(0.0263)$ & $1.9322(0.0274)$ & $1.9555(0.0252)$ \\
& $\alpha=2$ & $1.9687(0.0243)$ & $1.9771(0.0235)$ & $1.9886(0.0231)$ \\
\hline
\end{tabular}

Table 2. Estimated average lengths of the shape parameter and MSE of Weibull distribution.

\begin{tabular}{|c|c|c|c|c|}
\hline Size & Estimators & MLE & $\mathrm{BJ}$ & $\mathrm{BM}$ \\
\hline \multirow{4}{*}{25} & $\alpha=0.5$ & $0.5618(0.0931)$ & $0.4373(0.0943)$ & $0.5413(0.0911)$ \\
\hline & $\alpha=1$ & $0.9418(0.0893)$ & $0.9411(0.0902)$ & $0.9512(0.0882)$ \\
\hline & $\alpha=1.5$ & $1.4418(0.0878)$ & 1.4489 (0.0888) & 1.4558 (0.0863) \\
\hline & $\alpha=2$ & $1.9418(0.0860)$ & $1.9510(0.0851)$ & $1.9566(0.0846)$ \\
\hline \multirow{4}{*}{50} & $\alpha=0.5$ & $0.5511(0.0901)$ & $0.4411(0.0923)$ & 0.5215 (0.0897) \\
\hline & $\alpha=1$ & $0.9580(0.0881)$ & $0.9444(0.0896)$ & $0.9613(0.0877)$ \\
\hline & $\alpha=1.5$ & $1.4523(0.0867)$ & $1.4498(0.0875)$ & 1.4619 (0.0855) \\
\hline & $\alpha=2$ & 1.9545 (0.0855) & 1.9611 (0.0849) & 1.9662 (0.0831) \\
\hline \multirow{4}{*}{100} & $\alpha=0.5$ & $0.5429(0.0890)$ & 0.4489 (0.0896) & $0.5201(0.0884)$ \\
\hline & $\alpha=1$ & $0.9666(0.0873)$ & $0.9548(0.0887)$ & 0.9698 (0.0863) \\
\hline & $\alpha=1.5$ & $1.5318(0.0852)$ & $1.4517(0.0871)$ & $1.4819(0.0844)$ \\
\hline & $\alpha=2$ & $2.0418(0.0843)$ & 1.9718 (0.0839) & 1.9808 (0.0812) \\
\hline
\end{tabular}


Metropolis-Hastings algorithm (BM) is better compare to the others, moreover, Bayesian with help from Lindley's approximation is better than MLE estimators when $\alpha=2$. When the number of sample size increases the mean squared error (MSE) decreases in all cases.

In Table 2, the estimate of the shape parameter $\alpha$ of Weibull distribution with interval-censored data was compared between maximum likelihood (MLE), Bayesian using Lindley's approximation (BL) and Bayesian using Metropolis-Hastings algorithm (BM) by mean squared error (MSE). We found that the Bayesian using Metropolis-Hastings algorithm (BM) is the best compare to the others, moreover, Bayesian using Lindley's approximation is better than MLE estimators when $\alpha=2$. When the number of sample size increases the mean squared error (MSE) decreases in all cases.

\section{Conclusion}

The Bayesian using Metropolis-Hastings algorithm for estimating the scale and shape parameters of Weibull distribution-based interval-censored data is the best compare to others. In this paper, we contribute applying Bayesian estimation approach based on interval-censored data considered with Bayes using Makov Chain Monte Carlo (MCMC).

\section{References}

[1] Cohen, C.A. and Whitten, B. (1982) Modified Maximum Likelihood and Modified Moment Estimators for the ThreeParameter Weibull Distribution. Communications in Statistics-Theory and Methods, 11, 2631-2656. http://dx.doi.org/10.1080/03610928208828412

[2] Sinha, S.K. (1986) Bayes Estimation of the Reliability Function and Hazard Rate of a Weibull Failure Time Distribution. Trabajos de Estadística, 1, 47-56. http://dx.doi.org/10.1007/BF02863554

[3] Smith, R.L. and Naylor, J. (1987) A Comparison of Maximum Likelihood and Bayesian Estimators for the Three-Parameter Weibull Distribution. Applied Statistics, 36, 358-369. http://dx.doi.org/10.2307/2347795

[4] Singh, U., Gupta, P.K. and Upadhyay, S. (2002) Estimation of Exponentiated Weibull Shape Parameters under LINEX Loss Function. Communications in Statistics-Simulation and Computation, 31, 523-537. http://dx.doi.org/10.1081/SAC-120004310

[5] Hossain, A.M. and Zimmer, W.J. (2003) Comparison of Estimation Methods for Weibull Parameters: Complete and Censored Samples. Journal of Statistical Computation and Simulation, 73, 145-153. http://dx.doi.org/10.1080/00949650215730

[6] Nassar, M. and Eissa, F.H. (2005) Bayesian Estimation for the Exponentiated Weibull Model. Communications in Statistics-Theory and Methods, 33, 2343-2362. http://dx.doi.org/10.1081/STA-200031447

[7] Soliman, A.A., Abd Ellah, A.H. and Sultan, K.S. (2006) Comparison of Estimates Using Record Statistics from Weibull Model: Bayesian and Non-Bayesian Approaches. Computational Statistics \& Data Analysis, 51, 2065-2077. http://dx.doi.org/10.1016/j.csda.2005.12.020

[8] Kantar, Y.M. and Senoglu, B. (2008) A Comparative Study for the Location and Scale Parameters of the Weibull Distribution with Given Shape Parameter. Computers Geosciences, 34, 1900-1909. http://dx.doi.org/10.1016/j.cageo.2008.04.004

[9] Gupta, A., Mukherjee, B. and Upadhyay, S. (2008) Weibull Extension Model: A Bayes Study Using Markov Chain Monte Carlo Simulation. Reliability Engineering \& System Safety, 93, 1434-1443. http://dx.doi.org/10.1016/j.ress.2007.10.008

[10] Kundu, D. and Howlader, H. (2010) Bayesian Inference and Prediction of the Inverse Weibull Distribution for Type-II Censored Data. Computational Statistics \& Data Analysis, 54, 1547-1558. http://dx.doi.org/10.1016/j.csda.2010.01.003

[11] Pandey, B., Dwividi, N. and Pulastya, B. (2011) Comparison between Bayesian and Maximum Likelihood Estimation of the Scale Parameter in Weibull Distribution with Known Shape under Linex Loss Function. Journal of Scientific Research, 55, 163-172.

[12] Flygare, M.E. and Buckwalter, J.A. (1985) Maximum Likelihood Estimation for the 2-Parameter Weibull Distribution Based on Interval-Data. IEEE Transactions on Reliability, 34, 57-60. http://dx.doi.org/10.1109/TR.1985.5221930

[13] Al Omari, M.A., Ibrahim, N.A., Arasan, J. and Adam, M.B. (2012) Extension of Jeffreys’s Prior Estimate for Weibull Censored Data Using Lindley’s Approximation. Australian Journal of Basic and Applied Sciences, 5, 884-889.

[14] Hastings, W.K. (1970) Monte Carlo Sampling Methods Using Markov Chains and Their Applications. Biometrika, 57, 97-109. http://dx.doi.org/10.1093/biomet/57.1.97 
[15] Soliman, A.A., Abd-Ellah, A.H., Abou-Elheggag, N.A. and Ahmed, E.A. (2011) Modified Weibull Model: A Bayes Study Using MCMC Approach Based on Progressive Censoring Data. Reliability Engineering \& System Safety, 100, 48-57. http://dx.doi.org/10.1016/j.ress.2011.12.013

[16] Al Omari, M.A., Ibrahim, N.A., Arasan, J. and Adam, M.B. (2012) Bayesian Survival and Hazard Estimate for Weibull Censored Time Distribution. Journal of Applied Sciences, 12, 1313-1317. http://dx.doi.org/10.3923/jas.2012.1313.1317 
Scientific Research Publishing (SCIRP) is one of the largest Open Access journal publishers. It is currently publishing more than 200 open access, online, peer-reviewed journals covering a wide range of academic disciplines. SCIRP serves the worldwide academic communities and contributes to the progress and application of science with its publication.

Other selected journals from SCIRP are listed as below. Submit your manuscript to us via either submit@scirp.org or Online Submission Portal.
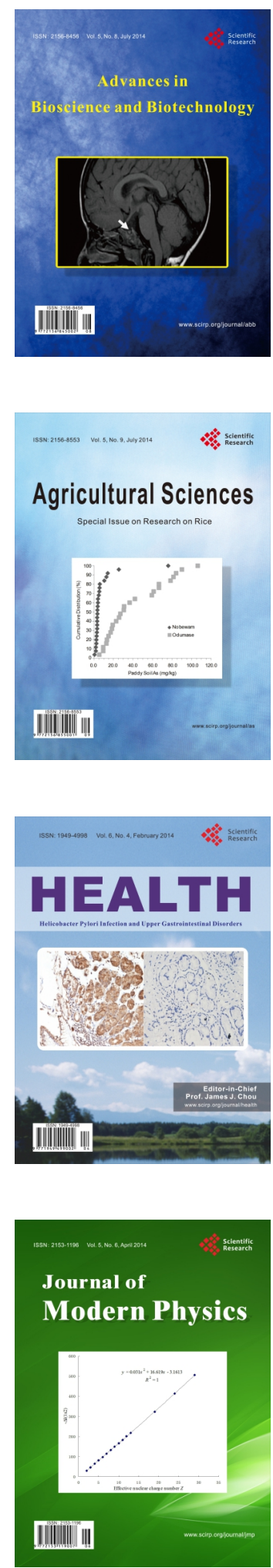
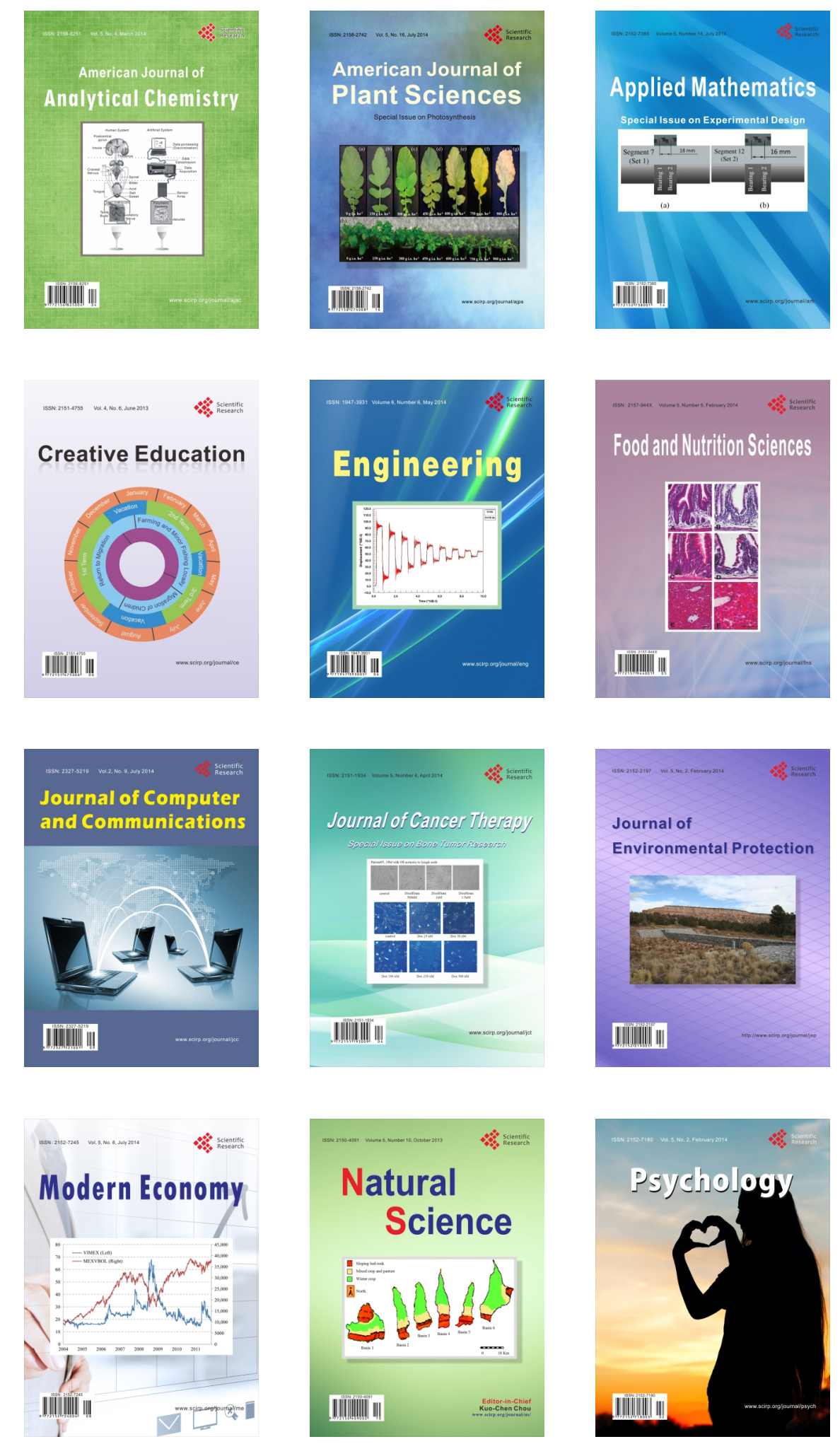